\title{
Características obstétricas de gestações em mulheres de 35 anos ou mais, segundo as
}

\section{regiões brasileiras}

\author{
Obstetric characteristics of pregnancies in women 35 years old or more, according brazilian regions \\ Características obstétricas del embarazo en mujeres de 35 años o más, según las regiones brasileñas
}

Recebido: 04/01/2022 | Revisado: 09/01/2022 | Aceito: 14/01/2022 | Publicado: 15/01/2022

Nathalie Araldi Piasson

ORCID: https://orcid.org/0000-0002-8553-3898

Universidade Comunitária da Região de Chapecó, Brasil

tnathaliepiasson.np@gmail.com

Gustavo Henrique Andrade

ORCID: https://orcid.org/0000-0001-9620-294X

Universidade Comunitária da Região de Chapecó, Brasil gustavo.andrade@unochapeco.edu.br

Mariana Stragliotto

ORCID: https://orcid.org/0000-0002-4593-8372

Universidade Comunitária da Região de Chapecó, Brasil mari_stragliotto@outlook.com

Lucimare Ferraz

ORCID: https://orcid.org/0000-0002-2487-8614

Universidade Comunitária da Região de Chapecó, Brasil ferraz.lucimare@gmail.com

Junir Antonio Lutinski

ORCID: https://orcid.org/0000-0003-0149-5415

Universidade Comunitária da Região de Chapecó, Brasil junir@unochapeco.edu.br

\begin{abstract}
Resumo
Este estudo objetivou investigar as características obstétricas de gestações em mulheres com 35 anos ou mais, segundo as regiões brasileiras. Foram obtidos registros de nascidos vivos do Sistema de Informações sobre Nascidos Vivos (SINASC), compreendendo os anos 2007, 2011 e 2017. Foram analisadas as variáveis: regiões brasileiras, idade da mãe, estado civil da mãe, tipo de parto, duração da gestação, peso ao nascer e algumas anomalias. Foi observado que existe um padrão unânime em todas as regiões do Brasil em que o número de mulheres que gestaram com 35 anos ou mais vem aumentando. Quanto ao estado civil, a maioria dessas gestantes eram casadas, número que decresceu, bem como o número de solteiras, e aumentou o número de mulheres em união estável. Quanto ao tipo de parto, na maioria das regiões brasileiras, houve um aumento de partos cesárea em mulheres que gestaram com 35 anos ou mais e uma diminuição de partos vaginais. Em todas as regiões do Brasil houve aumento no número de nascimentos pré-termo nessas mulheres, comparando-se aos três anos. Quanto ao peso ao nascer dos bebês, destaca-se o crescimento do número de nascidos com menos de 500 gramas. Quanto às anomalias encontradas em bebês nascidos de gestações tardias, verificou-se tendência de aumento na frequência. Discorreu-se, portanto, que mulheres que gestam com 35 anos ou mais poderão desenvolver mais facilmente complicações durante o processo gestacional e destaca-se principalmente a importância de um pré-natal bem aplicado a essas gestantes.
\end{abstract}

Palavras-chave: Anomalia; Gravidez tardia; Prematuridade; Via de parto; SINASC.

\begin{abstract}
This study aimed to investigate the obstetric characteristics of pregnancies in women aged 35 or over, according to Brazilian regions. Records of live births were recorded in the Live Birth Information System (SINASC), comprising the years 2007, 2011 and 2017. The variables were analyzed: regions, mother's age, mother's Brazilian state, type of delivery, duration of pregnancy, birth weight and some anomalies. It was observed that there is a unanimous pattern in all regions of Brazil in which the number of women who gestated aged 35 or over has increased. As for marital status, most of these pregnant women were married, a number that decreased, as well as the number of single women, and the number of women in a stable relationship increased. As for the type of delivery, in most Brazilian regions, there was an increase in cesarean deliveries in women who gestated aged 35 or more and a decrease in vaginal deliveries. In all regions of Brazil there was an increase in the number of preterm births in these women, comparing the three years. As for the babies' birth weight, the growth in the number of babies born with less than 500 grams stands out. As for the anomalies found in babies born with late pregnancies, there was a tendency to increase in frequency. It was argued, therefore, that
\end{abstract}


women who are pregnant at 35 years of age or more developed complications more easily during the gestational process and the importance of prenatal care well applied to these pregnant women stands out.

Keywords: Anomaly; Late pregnancy; Prematurity; Mode of delivery; SINASC.

\section{Resumen}

Este estudio tuvo como objetivo investigar las características obstétricas de los embarazos en mujeres de 35 años y más, según regiones brasileñas. Los registros de nacidos vivos se obtuvieron del Sistema de Información de Nacimientos Vivos (SINASC), que cubren los años 2007, 2011 y 2017. Las variables analizadas fueron: regiones brasileñas, edad de la madre, estado civil de la madre, tipo de parto, duración del embarazo, peso al nacer y algunas anomalías. Se observó que existe un patrón unánime en todas las regiones de Brasil en el que el número de mujeres embarazadas de 35 años o más ha ido en aumento. En cuanto al estado civil, la mayoría de estas mujeres embarazadas estaban casadas, número que disminuyó, al igual que el número de mujeres solteras, y aumentó el número de mujeres en relación estable. En cuanto al tipo de parto, en la mayoría de las regiones brasileñas, hubo un aumento de los partos por cesárea en las mujeres embarazadas de 35 años o más y una disminución de los partos vaginales. En todas las regiones de Brasil, hubo un aumento en el número de partos prematuros en estas mujeres, en comparación con tres años. En cuanto al peso al nacer de los bebés, hay un aumento en el número de bebés que nacen con un peso inferior a 500 gramos. En cuanto a las anomalías encontradas en los bebés nacidos de embarazos tardíos, hubo una tendencia a un aumento en la frecuencia. Por lo tanto, se discutió que las mujeres que están embarazadas de 35 años o más pueden desarrollar más fácilmente complicaciones durante el proceso gestacional y se destaca la importancia de una atención prenatal bien aplicada a estas mujeres embarazadas.

Palabras clave: Anomalía; Embarazo tardío; Precocidad; Ruta de entrega; SINASC.

\section{Introdução}

O tema central deste estudo é gestação tardia, que ocorre na faixa etária materna de 35 anos ou mais como descrito por Andrade (2004). Gonçalves e Monteiro (2012) relatam que a ocorrência desse tipo de gravidez vem aumentando no mundo, principalmente em países desenvolvidos e/ou em desenvolvimento. Ainda, a gestação numa faixa etária mais avançada é preocupante, pois estudos têm evidenciado associação importante entre idade materna igual ou superior a 35 anos e resultados perinatais adversos, representados por maior risco de complicações maternas, fetais e no recém-nascido como descrito por Andrade (2014). Mulheres de 35 a 39 anos, por exemplo, correm risco duas a três vezes maior de mortalidade materna do que as mulheres com idade na casa dos 20 anos, e este risco é ainda mais acentuado para as mulheres com 40 anos ou mais como descrito por Berg (2003). Dessa maneira, propõe-se uma pesquisa para investigar esta questão, de quais são as características obstétricas de gestações em mulheres acima de 35 anos, segundo as regiões brasileiras.

Em virtude da mudança comportamental vivenciada pela mulher atual, com alteração dos seus perfis de vida e importantes modificações socioculturais, criou-se, além de uma diminuição nos índices de natalidade, uma tendência no adiamento da gravidez como descrito por Prysak (1995). No ano 2000, aproximadamente $10 \%$ de todos os nascimentos ocorreram em mulheres com 35 anos ou mais, e essa realidade está aumentando cada vez mais com o passar dos anos como descrito por Andrade (2004).

É importante salientar que essa mulher nem sempre está ciente dos riscos que uma gestação tardia pode apresentar como descrito por Santos (2009). É evidenciado associação importante entre idade materna igual ou superior a 35 anos e resultados perinatais adversos, além da maior associação de gestações tardias com a ocorrência de partos cesárea como descrito por Andrade (2004).

A partir disso, faz-se imprescindível a educação em saúde e o planejamento familiar: apresentar à mulher as complicações e os riscos de uma gestação depois dos 35 anos, tanto para ela como para o feto e/ou recém-nascido, e sua suscetibilidade a isso como descrito por Azevedo (2002). Sendo assim, a partir desse conhecimento, entende-se que ela poderá optar com consciência pelo momento em que quer ter um filho.

Frente à realidade atual vivenciada pela mulher, esse estudo teve como objetivos identificar a frequência de gestações em mulheres acima de 35 anos; comparar as características obstétricas das mulheres acima de 35 anos em relação às mulheres 
com gestações em idade mais jovem; caracterizar o desfecho obstétrico e gestacional dessas mulheres e; verificar a incidência de anomalias por tipos nos bebês.

\section{Metodologia}

Foi realizado um estudo do tipo descritivo, retrospectivo com delineamento de um estudo ecológico, em que os dados referem-se a grupos de pessoas e não a indivíduos, e a unidade de estudo é uma área geográfica, sendo nesse caso específico as regiões do Brasil. Em geral, envolve menos custos e menor demanda de tempo como descrito por Aragão (2011). Foi feita uma comparação entre as regiões brasileiras sobre as características obstétricas de gestações em mulheres acima de 35 anos.

Os estudos descritivos têm por objetivo determinar a distribuição de doenças ou condições relacionadas à saúde, segundo o tempo, o lugar e/ou as características dos indivíduos. Nos estudos ecológicos, compara-se a ocorrência da doença/condição relacionada à saúde e a exposição de interesse entre agregados de indivíduos (populações de países, regiões ou municípios, por exemplo) para verificar a possível existência de associação entre elas segundo Lima (2003)

A população em estudo correspondeu a todos os registros de nascidos vivos do Sistema de Informações sobre Nascidos Vivos (SINASC, 2013), dos anos 2007, 2011 e 2017. Foram incluídos todos os registros de nascidos vivos nos anos citados, presentes no Sistema de Informações sobre Nascidos Vivos (SINASC), sem critérios de exclusão.

A pesquisa foi realizada com dados contidos na Declaração de Nascidos Vivo (DNV), utilizando a base de dados secundária do Sistema de Informações sobre Nascidos Vivos (SINASC), do Departamento de Informática do Sistema Único de Saúde (DATASUS), através do programa Tabnet. Foram reunidas informações relativas aos nascimentos ocorridos em todo o território nacional. No site, na área da linha foi selecionado a região e na área da coluna foi selecionado o ano do nascimento, e no período foi selecionado o ano 2007, 2011 e 2017. Já, nas seleções disponíveis, foram selecionadas as variáveis: idade da mãe, selecionando todas as categorias, estado civil da mãe (solteira, casada ou união consensual), tipo de parto, incluindo vaginal e cesáreo, duração da gestação, incluindo nascidos pré-termo, a termo e pós termo, peso ao nascer, incluindo peso inviável, baixo peso e peso normal, e, anomalia, incluindo a ocorrência e os tipos. Essa coleta foi realizada nos meses de outubro e novembro de 2019.

Os dados foram organizados em forma de tabela no programa Microsoft ${ }^{\circledR}$ Excel. Em seguida, as análises dos dados foram realizadas por meio do software SPSS, versão 20, de frequência e descritiva (média, desvio padrão, mediana, mínimo e máximo). As variáveis qualitativas foram apresentadas por meio da frequência absoluta e relativa.

O projeto de pesquisa não foi submetido ao Comitê de Ética devido à natureza pública e administrativa dos dados, apesar disso as informações foram tratadas rigorosamente com confidencialidade, respeitando os critérios da beneficência e não maleficência da produção científica, conforme os preceitos éticos do Conselho Nacional de Ética em Pesquisa (CONEP).

\section{Resultados e Discussão}

Foram analisados 32.069 .590 nascimentos, e, desse total, 3.705 .774 foram nascimentos de gestantes com 35 anos ou mais. Verificou-se que em todas as cinco regiões brasileiras houve um aumento do percentual de partos de mulheres com 35 anos ou mais no período avaliado (Figura 1). 
Figura 1. Evolução percentual das gestações segundo a idade das mulheres gestantes nos anos de 2007, 2011 e 2017, segundo as regiões brasileiras.

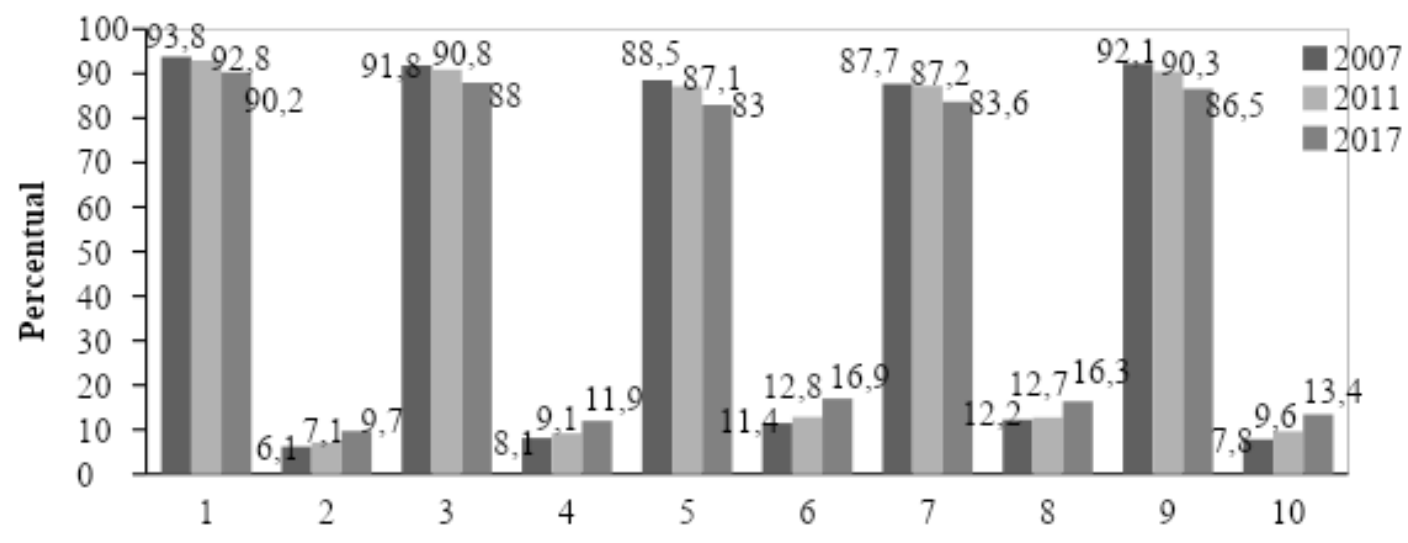

\section{Regiões brasileiras}

Fonte: Próprios autores (2021).

Quanto à variável Estado Civil, no ano de 2007, entre as mulheres com 35 anos ou mais, 55,6\% eram casadas, 43\% solteiras e 1,2\% encontravam-se em união consensual. No ano de 2011, às casadas eram de 52,2\%, solteiras 35,5\% e em união consensual 12,1\%. E em 2017, 52\% das mulheres com 35 anos mais eram casadas, 30\% solteiras e 17,7\% estavam em união consensual.

Em relação ao tipo de parto, no período analisado, nas regiões Norte e Nordeste, embora esteja crescendo o número de partos cesarianos, existe equilíbrio em comparação aos vaginais. Nas regiões Sul, Sudeste e Centro-Oeste dobram ou triplicam os partos cesarianos em relação aos vaginais (Figura 2). 
Figura 2. Evolução do tipo de parto optado por mulheres com 35 anos ou mais nos anos de 2007, 2011 e 2017, segundo as regiões brasileiras. PC: partos cesáreos; PV: partos vaginais.

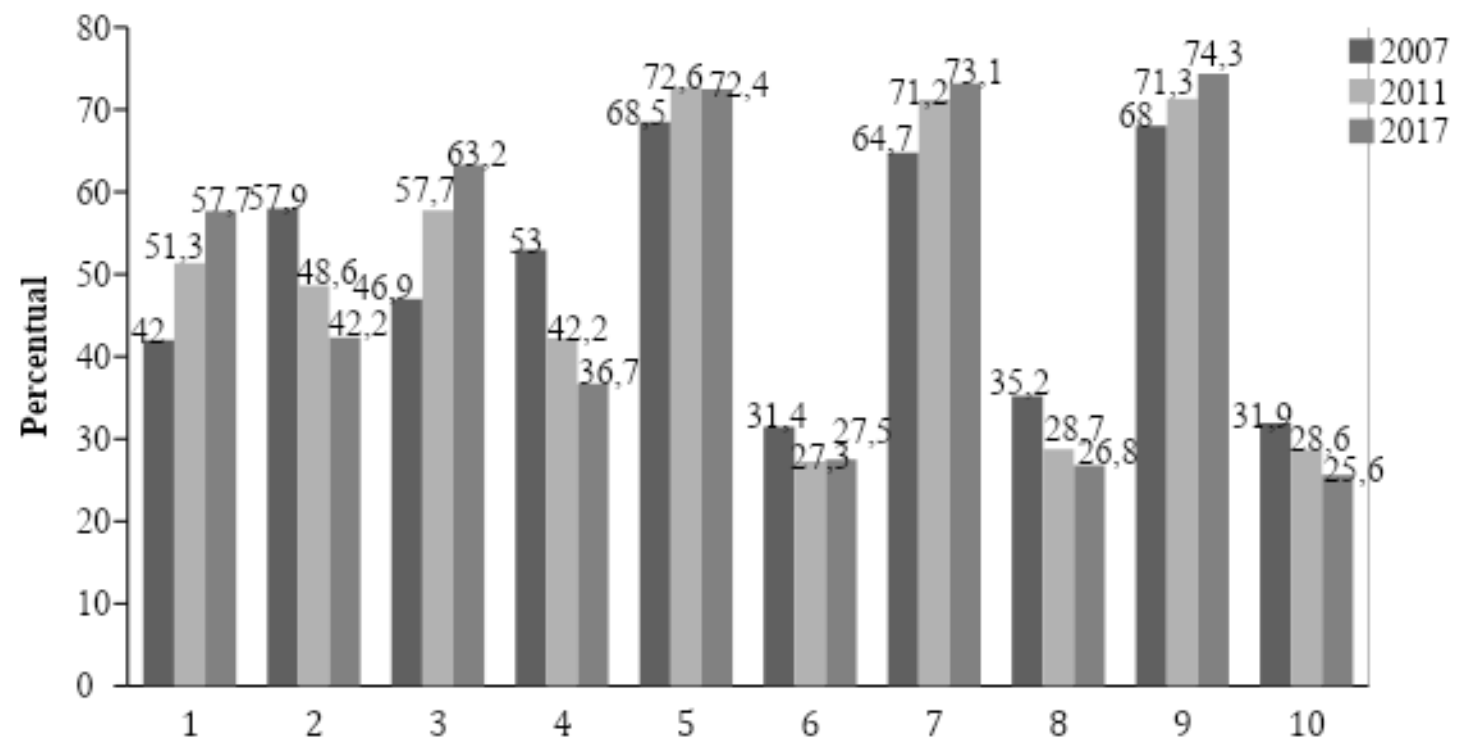

\section{Regiões brasileiras}

Fonte: Próprios autores (2021).

Foi percebida uma tendência de crescimento no número de partos prematuros, ou seja, antes de 36 semanas de gestação, nessas mulheres nos três anos analisados (Figura 3).

Figura 3. Evolução do percentual de mulheres com 35 anos ou mais que deram à luz com 22 até 36 semanas de gestação (gestação pré-termo) em cada ano analisado, segundo as regiões do Brasil.

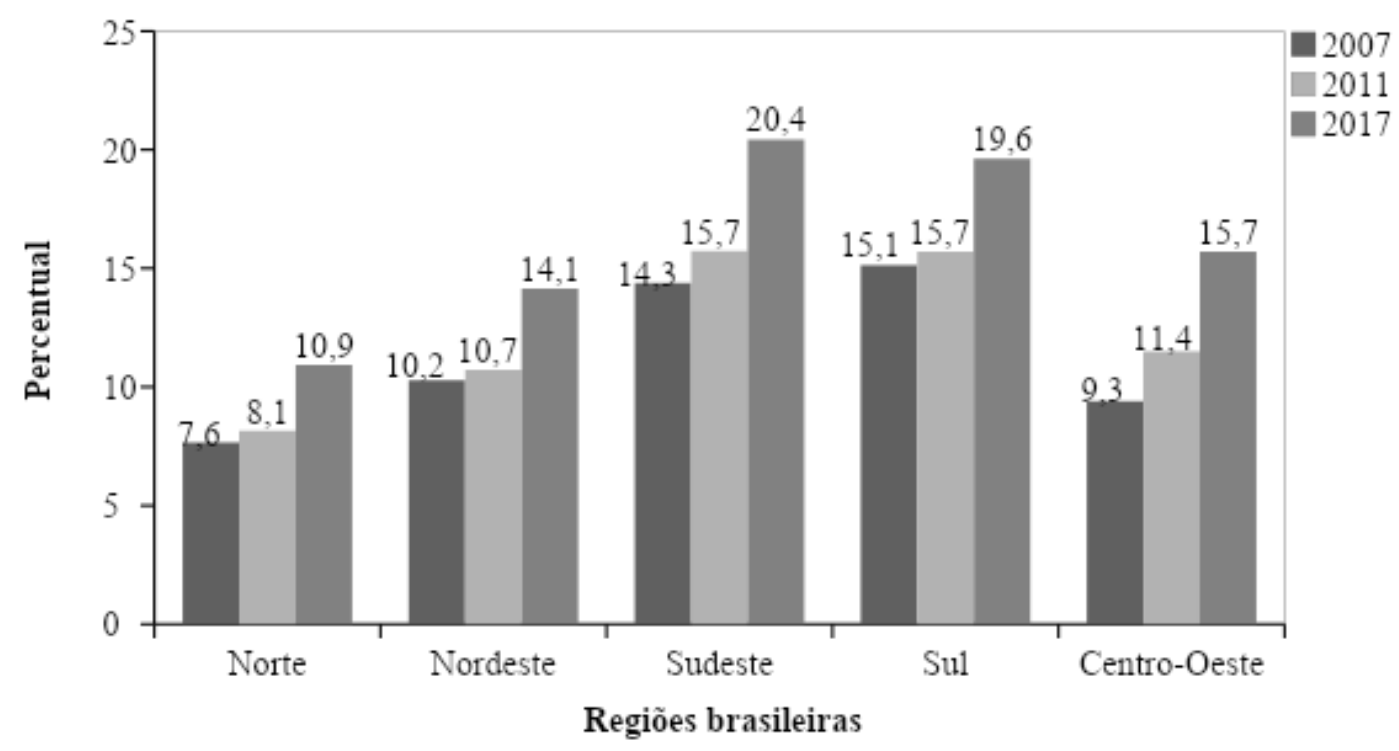

Fonte: Próprios autores (2021).

Em relação ao peso ao nascer dos bebês nascidos de gestações tardias no Brasil, no período analisado, destaca-se o crescimento do número de nascidos com menos de 500 gramas (Tabela 1). 
Tabela 1. Relação percentual segundo o peso ao nascer de bebês nascidos de mulheres com 35 anos ou mais nos anos de 2007 , 2011 e 2017, segundo as regiões brasileiras.

\begin{tabular}{|c|c|c|c|}
\hline \multirow[b]{2}{*}{ Regiões } & \multicolumn{3}{|c|}{ Peso ao nascer (\%) } \\
\hline & Menos $500 \mathrm{~g}$ & 500 a $2.499 \mathrm{~g}$ & $2.500 \mathrm{~g}$ ou mais \\
\hline \multicolumn{4}{|l|}{ Norte } \\
\hline 2007 & 0,09 & 8,04 & 91,87 \\
\hline 2011 & 0,12 & 7,01 & 92,86 \\
\hline 2017 & 0,11 & 8,45 & 91,44 \\
\hline \multicolumn{4}{|l|}{ Nordeste } \\
\hline 2007 & 0,12 & 9,20 & 90,68 \\
\hline 2011 & 0,19 & 9,51 & 90,31 \\
\hline 2017 & 0,19 & 9,42 & 90,40 \\
\hline \multicolumn{4}{|l|}{ Sudeste } \\
\hline 2007 & 0,08 & 11,22 & 88,70 \\
\hline 2011 & 0,11 & 11,10 & 88,79 \\
\hline 2017 & 0,12 & 10,68 & 89,20 \\
\hline \multicolumn{4}{|l|}{ Sul } \\
\hline 2007 & 0,06 & 11,03 & 88,91 \\
\hline 2011 & 0,07 & 10,74 & 89,18 \\
\hline 2017 & 0,11 & 10,10 & 89,80 \\
\hline \multicolumn{4}{|l|}{ Centro-Oeste } \\
\hline 2007 & 0,07 & 9,60 & 90,33 \\
\hline 2011 & 0,11 & 9,76 & 90,13 \\
\hline 2017 & 0,16 & 9,80 & 90,05 \\
\hline \multicolumn{4}{|l|}{ Total } \\
\hline 2007 & 0,09 & 10,36 & 89,55 \\
\hline 2011 & 0,12 & 10,38 & 89,50 \\
\hline 2017 & 0,13 & 10,07 & 89,80 \\
\hline
\end{tabular}

Fonte: Próprios autores (2021).

Quanto às anomalias congênitas, na região Norte, em 2007, o número foi de 8\%; em 2011, 9,1\%; em 2017, 13,2\%. Na região Nordeste, em 2007, 11,6\%; em 2011, 12,8\%; em 2017, 14,8\%. Na região Sudeste, em 2007, 15,9\%; em 2011, 16,8\%; em 2017, 22,8\%. Na região Sul, em 2007, 17,6\%; em 2011, 17,6\%; em 2017, 21,8\%. Na região Centro-Oeste, 9,9\% em 2007; em $2011,11 \%$ e em $2017,17,3 \%$.

Ainda, relacionando os tipos de anomalias encontrados em bebês nascidos de gestações tardias, de 2007 a 2017 , a maioria delas houve tendência de crescimento (Tabela 2). 
Tabela 2. Incidência por 100 mil nascidos vivos, segundo o tipo de anomalia em bebês nascidos de mulheres com 35 anos ou mais, segundo as regiões brasileiras, nos anos de 2007, 2011 e 2017. AEID: atresia/estenose de intestino delgado.

\begin{tabular}{|c|c|c|c|c|}
\hline \multirow[b]{2}{*}{ Regiões } & \multicolumn{4}{|c|}{ Anomalias ao nascer (x $100 \mathrm{mil})$} \\
\hline & Espinha bífida & Fenda labial & Cromossômica & AEID \\
\hline \multicolumn{5}{|l|}{ Norte } \\
\hline 2007 & 10,54 & 21,08 & 158,14 & \\
\hline 2011 & 18,40 & 78,19 & 156,37 & \\
\hline 2017 & 13,22 & 69,42 & 95,86 & 9,92 \\
\hline \multicolumn{5}{|l|}{ Nordeste } \\
\hline 2007 & 15,45 & 54,79 & 171,41 & \\
\hline 2011 & 14,09 & 55,09 & 134,52 & 1,28 \\
\hline 2017 & 29,63 & 43,94 & 124,66 & 2,04 \\
\hline \multicolumn{5}{|l|}{ Sudeste } \\
\hline 2007 & 25,90 & 60,43 & 212,69 & 3,92 \\
\hline 2011 & 23,82 & 66,01 & 178,29 & 2,72 \\
\hline 2017 & 34,23 & 64,37 & 156,33 & 2,04 \\
\hline \multicolumn{5}{|l|}{ Sul } \\
\hline 2007 & 20,26 & 69,77 & 283,59 & 6,75 \\
\hline 2011 & 24,91 & 87,18 & 257,38 & 6,23 \\
\hline 2017 & 23,06 & 75,35 & 161,45 & 7,69 \\
\hline \multicolumn{5}{|c|}{ Centro-Oeste } \\
\hline 2007 & 29,77 & 35,73 & 214,38 & 5,95 \\
\hline 2011 & 13,81 & 73,66 & 78,27 & \\
\hline 2017 & 18,30 & 51,86 & 103,72 & \\
\hline \multicolumn{5}{|l|}{ Total } \\
\hline 2007 & 21,52 & 56,31 & 209,84 & 3,23 \\
\hline 2011 & 20,50 & 67,82 & 170,98 & 2,52 \\
\hline 2017 & 28,70 & 60,71 & 141,34 & 3,32 \\
\hline
\end{tabular}

Fonte: Próprios autores (2021).

Historicamente, no mundo, existia uma divisão esclarecida de papéis: o homem era o provedor da casa, com autonomia para ditar regras e estabelecer as funções e a mulher era a cuidadora doméstica, detida aos afazeres desenvolvidos exclusivamente no lar como descrito por Fleck (2003). Ao longo das últimas décadas, houve a descaracterização desse padrão comportamental, de forma que a mulher contemporânea vem somando novas atividades ao seu estilo de vida, assumindo, por exemplo, espaço no mercado de trabalho, o que antes era praticamente só ocupado por homens, a fim de obter sua independência, estabilidade financeira e desenvolvimento intelectual. Presume-se que essas mudanças na dinâmica familiar possam ter sido influenciadas por fatores como a economia e a tecnologia, as quais se alteram constantemente ao longo do tempo como descrito por Fleck (2003).

Dessa forma, tal fato trouxe mudanças não apenas para a rotina da mulher contemporânea, mas também para seus projetos de vida e suas consequentes escolhas. Uma das áreas que sofreu um impacto importante em razão da multiplicidade de papéis assumidos pela mulher de hoje em dia é a maternidade. Em função de todas as demandas relacionadas à carreira e ao estudo, o projeto de ter filhos tem sido sistematicamente postergado como descrito por Lopes (2014). 
Nesse contexto, no Brasil, os resultados deste estudo apontam para uma tendência de aumento em todas as Regiões (Norte, Nordeste, Sudeste, Sul e Centro-Oeste), que mostra que as mulheres vêm, cada vez mais, adiando o processo gravídico. Observou-se um decréscimo, nos anos de 2007, 2011 e 2017, no percentual de mulheres que gestaram com menos de 35 anos e um aumento no percentual de mulheres com 35 anos ou mais. Isso pode ser explicado, por exemplo, pelo aumento de mulheres na participação do mercado de trabalho no período estudado, sendo que em 2007 a presença feminina representava 40,8\% do mercado formal. Já em 2016, esse número subiu para 44\%. Os dados são do Ministério do Trabalho e são baseados em pesquisas do Cadastro Geral de Emprego e Desemprego (CAGED) e da Relação Anual de Informações Sociais (RAIS).

Acompanhando esse aumento no número de mulheres se inserindo em diferentes áreas sociais e assumindo diferentes papeis, quanto à questão da gestação, especificamente a gestação tardia, ao ser analisado o estado civil destas mulheres, foi analisado que, nos três anos, o número de gestantes, tanto casadas quanto solteiras, com 35 anos ou mais, vem diminuindo, e quanto às mães em união consensual, este número vem crescendo. Apesar disso, nos três períodos, 2007, 2011 e 2017, a maioria delas eram casadas, ocupando cerca de 50\%. Sendo assim, associa-se o fato ao planejamento da gravidez, ou seja, à escolha de engravidar mais tardiamente. Em concomitância, destaca-se que a presença de um parceiro conjugal, estando esta gestante em um casamento ou em uma união consensual, pode constituir um atributo que a proteja contra possíveis efeitos adversos na gravidez, e pode servir como uma forte fonte de suporte emocional e afetivo conforme Souza (2016).

O nascimento de um filho é um momento de modificação na vida da mulher, e a resposta dessa mulher a estas mudanças é influenciada por diversos fatores, sendo estes individuais e ambientais. Destaca-se a importância do apoio de quem a cerca, especialmente do pai do bebê como descrito por Rapaport (2006).

Dependendo da qualidade do apoio que recebe, ou seja, da percepção que a mulher grávida tem de suporte social, isso se torna um possível fator protetor frente ao aparecimento de, por exemplo, sintomas depressivos ou de ansiedade. Estando a mulher em assistência afetiva, especialmente conjugal, em termos de satisfação, de apoio do parceiro e de proximidade do relacionamento, existe a correlação com a sintomatologia depressiva durante a gravidez e o pós-parto: mulheres com níveis de estresse altos durante a gestação ou que não possuem apoio e/ou um companheiro confidente são mais vulneráveis ao desenvolvimento da depressão pós-parto, por exemplo, conforme Pereira (1999).

Além da importância das questões psicológicas durante uma gravidez, este também é um processo em que praticamente todos os sistemas orgânicos da mulher passam por adaptações anatômicas, fisiológicas e bioquímicas, sendo que a maioria ocorre em resposta a estímulos fisiológicos a partir do feto e da placenta conforme Rezende (2007).

Schupp (2006) destaca que a idade ideal para se ter filhos está entre 20 e 29 anos, visto que é nessa faixa etária que são observados os melhores resultados maternos e perinatais. Sendo assim, cria-se um impasse: a tendência atual é de cada vez mais as mulheres adiarem o processo gravídico, ou seja, planejarem-se e optarem por uma gestação mais tardiamente. Para o Brasil (2012), gestantes com idade igual ou superior a 35 anos são consideradas tardias ou em idade avançada.

Ainda, os extremos da vida reprodutiva estão sempre ligados a complicações perinatais conforme Schupp (2006). Por esse motivo, a idade materna avançada causa preocupação do ponto de vista obstétrico. Isso seria consequência tanto da própria senescência ovariana quanto da frequência maior de doenças crônicas em mulheres nessa fase da vida conforme Azevedo (2002).

Partindo-se do pressuposto de que a mulher que gesta com 35 anos ou mais poderá desenvolver com mais facilidade organicamente complicações durante o processo gestacional e durante o parto, também é trazido o tipo de parto como um possível impasse. Mascarello (2017) destaca que as gestantes e os profissionais de saúde precisam ter conhecimento dos riscos maternos associados às diferentes vias de parto, utilizando as melhores evidências, sendo que o parto cesáreo, muitas vezes, é realizado sem indicação clínica.

Observou-se a partir dos resultados deste estudo uma tendência ao aumento no número de mulheres gestantes com idade igual ou superior a 35 anos que realizaram a cesariana nos anos de 2007, 2011 e 2017, bem como um decréscimo no número de 
partos vaginais, sendo que nas regiões Sul, Sudeste e Centro-Oeste dobram ou triplicam o número de partos cesarianos em relação aos partos vaginais.

Concomitantemente é reforçada a presença de infecção pós-parto, sendo maior nas cesáreas, bem como a necessidade de internação em UTI, e as hemorragias são mais graves do que no parto vaginal Segundo Mascarello (2017). Ou seja, nas regiões Norte, Nordeste, Sul e Centro-Oeste, as cesarianas devem ser realizadas com mais prudência e a grande questão relacionada a essa via de parto está em fazer bom uso desse procedimento que pode ser um importante recurso para a redução da mortalidade da mãe e do bebê, mas, por outro lado, quando usado de maneira excessiva e não indicada clinicamente, pode estar associado a um risco negativo e aumentado de resultados maternos graves, ainda mais em mulheres que já possuem disposição a complicações, como as com 35 anos ou mais.

Já a Região Sudeste foi a única que apresentou, apesar de discreto, um aumento no número de partos vaginais e um decréscimo no número de partos cesáreas comparando apenas 2011 e 2017. Segundo Melo (2015), o parto vaginal merece ser explicado e dialogado para com as gestantes, por meio de ações educativas durante o pré-natal, e também incentivado nas que estiverem saudáveis e aptas, tanto pelo fato de ser um processo natural, quanto por ser provedor de benefícios mútuos para a mãe e para o bebê. Além disso, o parto vaginal possui menos complicações do que o processo cirúrgico que é uma cesariana e possui uma recuperação mais rápida para a mulher.

Em seguida, tem-se outro impasse gerado pela gestação tardia: o nascimento prematuro. Para que um bebê desenvolva seus membros, tecidos e órgãos de forma adequada é necessário que o período de gestação, que vai da concepção até o nascimento, dure em média de 38 a 42 semanas. Chama-se parto a termo aquele que ocorre entre 37 e 42 semanas de gravidez. No entanto, pode acontecer de o bebê nascer antes: entre as entre 22 e 37 semanas incompletas de gestação. Esta situação é chamada de parto pré-termo, de acordo com a Organização Mundial de Saúde (OMS), e o bebê é considerado prematuro. Ainda, considera-se como pós-termo o nascimento que ocorre após 42 semanas de gestação. Ambos os casos são situações especiais, tanto os nascimentos pré-termo como os pós-termo, de modo que envolvem riscos para mãe e para a criança e também demandam maiores cuidados.

Este estudo mostrou que, em todas as regiões do Brasil e nos anos analisados, houve crescimento no percentual de mulheres com 35 anos ou mais que vieram a dar à luz com 22 a 36 semanas de gestação, ou seja, aumentou o número de nascidos pré-termo nessas mulheres. A região Sudeste foi a região na qual mais ficou evidente esse crescimento, passando de 15,7\% em 2011 para 20,4\% em 2017.

Entende-se que a prematuridade é uma das principais causas de morbidade e mortalidade a curto e longo prazo para o bebê, merecendo uma maior atenção a fim de preveni-la. Ele resulta de um conjunto de fatores interrelacionados e traz, anualmente, aos países um alto custo social e econômico, uma vez que impacta significativamente na mortalidade infantil e na qualidade de vida dos que sobrevivem com sequelas, conforme Assunção (2012).

A literatura destaca a importância do pré-natal como sendo um dos principais determinantes da adequada evolução gestacional, permitindo identificar situações de risco que podem acarretar o trabalho de parto prematuro precocemente. Dessa forma, tem-se uma maior garantia frente à saúde da mãe e do bebê, prevenindo complicações conforme Meme (2005).

Outro importante indicador de saúde de uma população e um relevante determinante biológico da sobrevivência do bebê recém-nascido em condições adversas é o peso baixo ao nascer. O recém-nascido abaixo de 1.000 gramas é classificado como extremo baixo peso, os que nascem com 1.000 gramas a 1.499 gramas são classificados como recém-nascidos de muito baixo peso e os com 1.500 gramas a 2.500 gramas são classificado como baixo peso como descrito pelo Ministério da Saúde (2012)

Neste estudo, ao ser analisada a relação percentual segundo o peso ao nascer dos bebês nascidos de gestações tardias, nos anos analisados, foi observado que houve aumento dos nascidos com menos de 500 gramas, ou seja, de bebês extremo baixo peso. Isso ocorreu de forma unânime entre as regiões brasileiras, com destaque para a região Centro-Oeste, que dobrou esse aumento entre 2007 e 2017. Ainda, neste mesmo período, também houve crescimento do percentual na maioria das regiões 
(Norte, Nordeste e Centro-Oeste) quanto ao peso ao nascer 500 a 2.499 gramas, sendo que duas regiões (Sudeste e Sul), em contrapartida, apresentaram decréscimo.

A importância que tem um bebê nascer com o peso abaixo do normal decorre da capacidade que apresenta para predizer risco de adoecimento nessas crianças nascidas sob essa condição, de modo que elas se encontram em maior risco de possuírem sequelas: convulsões e paralisia cerebral, surdez e cegueira conforme Rodrigues (2006). Além de, na idade escolar, estarem sujeitos a apresentar dificuldade de aprendizado, menor capacidade de funcionamento social, comportamental e adaptativo, mesmo que sem deteriorações no neurodesenvolvimento, quando comparadas a crianças que nasceram com o peso adequado descrito por Resegue (2008).

Ainda, essas condições de baixo peso, de muito baixo peso e de extremo baixo peso refletem uma exposição materna a fatores de risco, como condições de doenças na gestação e/ou certa deficiência em uma atenção ao pré-natal e no parto, sendo que peso abaixo do normal ao nascimento é o fator de risco isolado mais importante para a mortalidade infantil e é maior nos extremos de idade da mãe conforme Sass (2011).

Outra questão perinatal importante a ser considerada nas gestações em mulheres com idade mais avançada é a de o bebê estar mais predisposto a anomalias congênitas conforme Sass (2011). Define-se por anomalia congênita todo defeito funcional ou estrutural, presente no nascimento ou que se manifesta ao longo da vida, resultado de alterações que ocorrem durante o desenvolvimento embrionário, podendo ser encontrados desde pequenas assimetrias até defeitos com comprometimentos maiores estéticos ou funcionais conforme Melo (2010).

O aumento no número de gestações em mulheres com 35 anos ou mais analisado neste estudo também vem acompanhado de um aumento no número de bebês nascidos dessas mulheres com algum tipo de anomalia congênita. No período 2007, 2011 e 2017, em todas as regiões brasileiras houve crescimento nesses percentuais, sendo no Sudeste e no Centro-Oeste os números mais evidentes.

Melo (2010) diz que existe, sim, relação entre idade materna e a presença de anomalia congênita no nascimento, de modo que, para as mães com idade igual ou maior que 35 anos, as chances de o bebê ter anomalia congênita são de 11,4 vezes maior quando comparadas às mães jovens, com idade entre 20 a 34 anos. Sendo assim, este estudo especificou para análise quatro anomalias, disponibilizadas no site DATASUS/SINASC: espinha bífida; fenda labial; cromossômica; atresia/estenose de intestino delgado (AEID).

Quanto à espinha bífida, excetuando-se a região Centro-Oeste, em todas as demais regiões brasileiras houve aumento no número de bebês nascidos de mulheres com 35 anos ou mais com esta anomalia, nos anos analisados. Cunha et al. (2005) esclarece que pesquisadores já comprovaram que os extremos de idade materna são fatores de predisposição para a malformação em estudo, ou seja, que tanto mães bastante jovens (13 a 19 anos) quanto mães em idade mais avançada (35 anos ou mais) têm maiores chances de ter um bebê com espinha bífida.

Segundo Martelli et al. (2010), a idade materna é considerada fator de risco para diversas alterações cromossômicas, mas não encontrou um consenso de se ela representa ou não fator de risco para a anomalia fenda labial. Salientam que a maioria dos estudos até 1970 sugere associação entre a malformação e a idade materna, mas que a partir disso outros trabalhos vêm surgindo e os resultados são conflitantes, alguns apresentando relação e outros não. Neste estudo, analisando 2007, 2011 e 2017, em todas as regiões brasileiras o número de nascidos de gestações tardias com fenda labial sofreu aumento, com exceção da região Nordeste, e mostrou ser o segundo tipo de anomalia com maior incidência por 100 mil nascidos vivos.

Além disso, no mesmo período, um dado notório é que em todas as regiões do Brasil houve decréscimo no número de nascidos de gestantes tardias com anomalia cromossômica, apesar de, ainda assim, ser a anomalia, entre as quatro, com maior incidência por 100 mil nascidos vivos. Cosme et al. (2017) relataram que existe uma predisposição em se ter um número maior de nascidos portadores de anomalias congênitas, sobretudo de anomalias cromossômicas, em mulheres nos dois extremos de 
idade reprodutiva. Segundo ele, entre as cromossomopatias, a mais encontrada foi a Síndrome de Down e a idade materna avançada é um dos fatores envolvidos nesse resultado, pois está associada à maior incidência de aneuploidias.

A atresia de intestino delgado é uma importante causa de obstrução gastrintestinal, com alta taxa de morbidade em recém-natos e ela também está frequentemente associada a anomalias cromossômicas conforme Figuerêdo (2005). Os números também foram de crescimento entre os nascidos de mulheres com 35 anos ou mais sob condição de atresia/estenose de intestino delgado (AEID), evidenciando a região Norte que, em 2017, com 9,92 de incidência por 100 mil nascidos vivos, se sobressaiu frente às demais: Nordeste e Sudeste, por exemplo, contavam com 2,04 e aproximando-se apenas da região Sul, com 7,69.

Sendo assim, as anomalias congênitas associam-se à morbimortalidade infantil, principalmente no período neonatal, tornando-se importante o seu diagnóstico precoce para o planejamento e a alocação de recursos dos serviços de saúde especializados (pré- natal, natal e pós-natal), para a redução da morbimortalidade, principalmente neonatal precoce, e para a melhora da qualidade de vida e dos índices de sobrevida conforme Cosme (2017). O pré-natal faz a detecção precoce e é de extrema importância ao evitar evolução e sequelas da doença. No pós-natal o acompanhamento evita a complicação da doença conforme Melo (2010).

\section{Conclusão}

Frente aos objetivos deste trabalho, foi concluído que, no Brasil, num período de dez anos, sendo analisados os anos de 2007, 2011 e 2017, as mulheres, estão postergando cada vez mais o processo gravídico, ou seja, o número de gestações em mulheres com 35 anos ou mais está em constante crescente no país.

Sugere-se o planejamento familiar frente a um processo gravídico e, em especial nessas mulheres, que, muitas vezes escolhem ter o filho mais tardiamente, visto a sua maior predisposição a complicações obstétricas. E junto a isso, a questão de se ter um parceiro faz-se importante. Neste estudo, frente ao crescente número de essas gestantes em união estável, concluímos que o meio, e o cônjuge em especial, servem como uma forte fonte de suporte emocional e afetivo, além de poderem constituir um atributo que a proteja contra possíveis efeitos adversos na gravidez. Sugere-se estudos de como o planejamento familiar pode alterar possíveis desfechos.

Ainda, o comportamento de postergar a gestação traz consigo questões imprescindíveis do ponto de vista obstétrico, sendo que as gestações consideradas tardias apresentam risco aumentado de complicações maternas e fetais. A via de parto é um fator relevante ao fato, em vista de que as mulheres que gestam no extremo superior da sua idade reprodutiva têm trazido consigo a tendência de realizar mais partos cesarianos do que vaginais. Salienta-se que o primeiro pode estar associado não apenas e muitas vezes à escolha da mãe, mas porque ela apresenta complicações de uma gestação tardia que não a permite realizar um parto mais natural como é o vaginal.

Encaixam-se, assim, alguns levantamentos de questões de saúde pública. Os gastos com as cesarianas, por exemplo, são superiores aos de partos vaginais, e isso principalmente caso ela venha a precisar de um leito na UTI, por complicações às quais ela está ainda mais sujeita pelo fato de estar realizando um parto cesáreo, como hemorragias ou infecções. Ainda, a gestante tardia está mais predisposta a desenvolver alguma doença durante o processo gravídico do que as gestantes mais jovens, então outra questão são as menores taxas de sucesso frente a esta gestação em uma atenção primária de saúde, sendo necessário que a gestante tardia seja encaminhada aos centros especializados de saúde obstétrica.

Outro fator relevante considerando a gestação que ocorre tardiamente, frente às suas complicações e desfechos, é o nascimento de bebês prematuros, situação crescente entre as mulheres gestantes com 35 anos ou mais nos anos analisados por este estudo. Isso é impactado diretamente pela qualidade do pré-natal que essa gestante recebe, que pode identificar situações de risco que levem ao trabalho de parto antes do período normal. 
Além disso, tanto o pré-natal como a boa assistência no trabalho de parto também se fazem imprescindíveis frente à resolubilidade de complicações como o baixo peso ao nascer, crescente entre as mulheres gestantes tardias deste estudo. Junto à prematuridade, o baixo peso ao nascer encontra-se entre as principais causas de morbidade e mortalidade infantis, podendo ser mais bem manejado quando o sistema de saúde está preparado para atender a uma gestante mais predisposta a doenças e complicações como é a tardia, bem como para atender uma criança que nasce com um peso bastante abaixo do ideal, predisposta ao adoecimento e sequelas, seja via profissionais bem capacitados, seja via infraestrutura adequada para atender esses pacientes.

Este estudo também mostrou haver uma relação entre a idade materna avançada e o acometimento de anomalia congênita no bebê. A grande maioria das anomalias (espinha bífida, fenda labial, AEID) mostraram-se crescentes entre as gestantes tardias, com exceção da anomalia cromossômica. Apesar disso, esta é a de maior incidência por 100 mil nascidos vivos nas regiões brasileiras. Traz-se, novamente, a questão da importância de um pré-natal bem aplicado às gestantes, visto que ele pode diagnosticar precocemente uma anomalia e, assim, reduzir a mortalidade e favorecer qualidade de vida no bebê.

\section{Referências}

Andrade, P. C., Linhares, J. J., Martinelli, S., Antonini, M., Lippi, U. G., \& Baracat, F. F. (2004). Resultados perinatais em grávidas com mais de 35 anos: estudo controlado. Revista Brasileira de Ginecologia E Obstetrícia, 26(9), 697-701. https://doi.org/10.1590/s0100-72032004000900004

Aragão, J. (2013). Introdução aos estudos quantitativos utilizados em pesquisas científicas. Revista Práxis, 3(6). https://doi.org/10.25119/praxis-3-6-566

Assunção, P. L., Novaes, H. M. D., Alencar, G. P., Melo, A. S. de O., \& Almeida, M. F. de. (2012). Fatores associados ao nascimento pré-termo em Campina Grande, Paraíba, Brasil: um estudo caso-controle. Cadernos de Saúde Pública, 28(6), 1078-1090. https://doi.org/10.1590/s0102-311x2012000600007

Azevedo, G. D. de, Freitas Júnior, R. A. de O., Freitas, A. K. M. S. de O., Araújo, A. C. P. F. de, Soares, E. M. M., \& Maranhão, T. M. de O. (2002). Efeito da Idade Materna sobre os Resultados Perinatais. Revista Brasileira de Ginecologia E Obstetrícia, 24(3). https://doi.org/10.1590/s0100-72032002000300006

Berg, C. (2003). Pregnancy-related mortality in the United States, 1991-1997. Obstetrics \& Gynecology, 101(2), 289-296. https://doi.org/10.1016/s00297844(02)02587-5

Bussâmara Neme. (2006). Obstetrícia básica. Sarvier.

Brasil (2011), Ministério da Saúde. Secretaria de Atenção à Saúde. Departamento de Ações Programáticas Estratégicas. Atenção à saúde do recém-nascido: guia para profissionais da saúde. Cuidados com o recém-nascido pré-termo.

Brasil, Ministério da Saúde, Secretaria de Atenção à Saúde, \& Departamento de Ações Programáticas Estratégicas. (2012). Gestação de alto risco: manual técnico. Brasil (2020), Ministério do Trabalho. Programa de disseminação das estatísticas do trabalho (PDET) - CAGED e RAIS. Disponível em: http://pdet.mte.gov.br/ Brasil (2019), Sistema Nacional de Nascidos Vivos (SINASC). Nascidos Vivos. http://tabnet.datasus.gov.br/cgi/deftohtm.exe?sinasc/cnv/nvuf.def.

Cosme, H. W., Lima, L. S., \& Barbosa, L. G. (2017). Prevalência de anomalias congênitas e fatores associados em recém nascidos do município de São Paulo no período de 2010 a 2014. Revista Paulista de Pediatria, 35(1), 33-38. https://doi.org/10.1590/1984-0462/;2017;35;1;00002

Cunha, C. de J. da, Fontana, T., Garcias, G. de L., \& Martino-Roth, M. da G. (2005). Fatores genéticos e ambientais associados a espinha bífida. Revista Brasileira de Ginecologia E Obstetrícia, 27(5). https://doi.org/10.1590/s0100-72032005000500007

Figueirêdo, S. da S., Ribeiro, L. H. V., Nóbrega, B. B. da, Costa, M. A. B., Oliveira, G. L., Esteves, E., Monteiro, S. S., \& Lederman, H. M. (2005). Atresia do trato gastrintestinal: avaliação por métodos de imagem. Radiologia Brasileira, 38(2), 141-150. https://doi.org/10.1590/s0100-39842005000200011।

Fleck, A. C., \& Wagner, A. (2003). A mulher como a principal provedora do sustento econômico familiar. Psicologia Em Estudo, 8(spe). https://doi.org/10.1590/s1413-73722003000300005

Gonçalves, Z. R., \& Monteiro, D. L. M. (2012). Complicações maternas em gestantes com idade avançada. Femina. 40(5), 275-279

Lima-Costa, M. F., \& Barreto, S. M. (2003). Tipos de estudos epidemiológicos: conceitos básicos e aplicações na área do envelhecimento. Epidemiologia e serviços de saúde, 12(4), 189-201.

Lopes, M. N., Dellazzana-Zanon, L. L., \& Boeckel, M. G. (2014). A multiplicidade de papéis da mulher contemporânea ea maternidade tardia. Temas em psicologia, 22(4), 917-928.

Martelli, D. R. B., Cruz, K. W. D., Barros, L. M. D., Silveira, M. F., Swerts, M. S. O., \& Martelli Júnior, H. (2010). Avaliação da idade materna, paterna, ordem de paridade e intervalo interpartal para fissura lábio-palatina. Brazilian Journal of Otorhinolaryngology, 76, $107-112$.

Mascarello, K. C., Horta, B. L., \& Silveira, M. F. (2017). Complicações maternas e cesárea sem indicação: revisão sistemática e meta-análise. Revista de Saúde Pública, 51, 105. 
Research, Society and Development, v. 11, n. 1, e57611125334, 2022

(CC BY 4.0) | ISSN 2525-3409 | DOI: http://dx.doi.org/10.33448/rsd-v11i1.25334

de Melo, J. K. F., Davim, R. M. B., \& da Silva, R. R. A. (2015). Vantagens e desvantagens do parto normal e cesariano: opinião de puérperas. Revista de Pesquisa Cuidado é Fundamental Online, 7(4), 3197-3205.

de Melo, W. A., Zurita, R. C. M., Uchimura, T. T., \& Marcon, S. S. (2010). Anomalias congênitas: fatores associados à idade materna em município sul brasileiro, 2000 a 2007. Revista eletrônica de enfermagem, 12(1).

Pereira, M. G., Santos, A. C., \& Ramalho, V. (1999). Adaptação à gravidez: Um estudo biopsicossocial. (3), 583-590,

Prysak, M. (1995). Pregnancy Outcome in Nulliparous Women 35 Years and Older. Obstetrics \& Gynecology, 85(1), 65-70. https://doi.org/10.1016/00297844(94)00330-g

Rapoport, A., \& Piccinini, C. A. (2006). Apoio social e experiência da maternidade. Journal of Human Growth and Development, 16(1), 85. https://doi.org/10.7322/jhgd.19783

Resegue, R., Puccini, R. F., \& Silva, E. M. K. D. (2008). Risk factors associated with developmental abnormalities among high-risk children attended at a multidisciplinary clinic. Sao Paulo Medical Journal, 126(1), 4-10.

Rezende Filho, J., \& Montenegro, C. A. B. (2007). Obstetrícia fundamental. (11a ed.).

de Rodrigues, M. C., Mello, R. R., \& Fonseca, S. C. (2006). Dificuldade de aprendizagem em escolares de muito baixo peso ao nascer. Jornal de Pediatria, 82, 6-14.

Santos, G. H. N. D., Martins, M. D. G., Sousa, M. D. S., \& Batalha, S. D. J. C. (2009). Impacto da idade materna sobre os resultados perinatais e via de parto. Revista Brasileira de Ginecologia e Obstetrícia, 31, 326-334.

Sass, A., Gravena, A. A. F., Pelloso, S. M., \& Marcon, S. S. (2011). Resultados perinatales en los extremos de la vida reproductiva y factores asociados al bajo peso al nacer. Revista Gaúcha de Enfermagem, 32(2), 362-368.

Schupp, T. R. (2006). Gravidez após os 40 anos de idade: análise dos fatores prognósticos para resultados maternos e perinatais diversos (Doctoral dissertation, Universidade de São Paulo).

Souza, W. P. D. S., Maia, E. M. C., Oliveira, M. A. M., Morais, T. I. S., Cardoso, P. S., Lira, E. C. S. D., \& Melo, H. M. D. A. (2016). Gravidez tardia: relações entre características sociodemográficas, gestacionais e apoio social. Boletim de psicologia, 66(144), 47-59. 